\title{
RETRACTED ARTICLE: Medical image encryption technique in big media environment
}

\author{
Xia Han ${ }^{1} \cdot$ Chao Xiong $^{1} \cdot$ Yuan $\mathrm{Li}^{1} \cdot$ FengYou He$^{1} \cdot$ \\ Hongwei Du ${ }^{1}$
}

Received: 18 December 2017 /Revised: 24 December 2017 / Accepted: 28 December $2017 /$ Published online: 20 February 2018

(C) Springer Science+Business Media, LLC, part of Springer Nature 2018

The Editor-in-Chief has retracted this article [1], which was published as part of special issue "Multi-source Weak Data Management using Big Data", because its content has been duplicated from an unpublished manuscript authored by Priya Selvam and Santhi Balachandran without permission. In addition, there is evidence suggesting authorship manipulation and an attempt to subvert the peer review process.

Author Chao Xiong agrees to this retraction. Authors Xia Han, Yuan Li, FengYou He, and Hongwei Du have not responded to correspondence about this retraction.

\section{References}

1. Han, X., Xiong, C., Li, Y. et al. Medical image encryption technique in big media environment. Multimed Tools Appl (2018). https://doi.org/10.1007/s11042-017-5598-5

Electronic supplementary material The online version of this article (https://doi.org/10.1007/s11042-0175598-5) contains supplementary material, which is available to authorized users.

Chao Xiong

xiongchao1778@126.com

1 School of Electrical and Photoelectronic Engineering, Changzhou Institute of Technology,

Changzhou, Jiangsu Province 213032, China 